\title{
AN ANALOGUE OF BÄCKLUND'S THEOREM IN AFFINE GEOMETRY
}

\author{
ShiIng-Shen Chern* and ChuU-Lian Terng**
}

\begin{abstract}
It is well-known that there is a correspondence between solutions of the Sine-Gordon equation (SGE)

$$
\frac{\partial^{2} \phi}{\partial x^{2}}-\frac{\partial^{2} \phi}{\partial t^{2}}=\sin \phi
$$

and the surfaces of constant curvature -1 in $\mathbf{R}^{3}$ (see below). The classical Bäcklund transformation of such surfaces furnishes a way to generate new solutions of the SGE from a given solution. This has received much attention in recent studies of the soliton solutions of the SGE, and the technique has been used successfully in the study of other non-linear evolution equations. In the first section of this paper we present a simple derivation of the classical Bäcklund theorem and its applications by using the method of moving frames.

Our main result concerns affine minimal surfaces. They arise as the solution of the variation problem for affine area. The corresponding Euler-Lagrange equation is a fourth order partial differential equation. In §2, we develop the basic properties of affine minimal surfaces.In $\$ 3$ we study the transformation of affine surfaces by realizing them as the focal surfaces of a line congruence. The natural conditions that the congruence be a W-congruence and that the affine normals at corresponding points be parallel lead to the conclusion that both surfaces are affine minimal. This is the content of Theorem 4, the main result of our paper. As in the classical case, the Theorem leads to the construction of new affine minimal surfaces from a given one by the solution of a completely integrable system of first order partial differential equations.
\end{abstract}

1. The classical Bäcklund theorem and its consequences. Let $M$ be a surface in $\mathbf{R}^{3}$. We choose a local field of orthonormal frames $v_{1}, v_{2}, v_{3}$ with origin $X$ in $\mathbf{R}^{3}$ such that $X$ is a point of $M$ and the vectors $v_{1}, v_{2}$ are tangent to $M$ at $X$. Let $\theta_{1}, \theta_{2}, \theta_{3}$ be the dual coframe of $v_{1}, v_{2}, v_{3}$. We can write

$$
\begin{aligned}
d x & =\sum_{\alpha} \theta_{\alpha} v_{\alpha} \\
d v_{\alpha} & =\sum_{\beta} \theta_{\alpha \beta} v_{\beta},
\end{aligned}
$$

Here and throughout this paper we shall agree on the index ranges

$$
1 \leqq i, j, k \leqq 2, \quad 1 \leqq \alpha, \beta, \gamma \leqq 3 .
$$

The structure equations of $\mathbf{R}^{3}$ are

*Work done under partial support of NSF grant MCS74-23180.

**Work done under partial support of NSF Grant MCS76-01692.

Received by the editors on April 13, 1978.

Copyright (c) 1980 Rocky Mountain Mathematics Consortium 


$$
\begin{aligned}
& d \theta_{\alpha}=\sum_{\beta} \theta_{\beta} \wedge \theta_{\beta \alpha}, \theta_{\alpha \beta}+\theta_{\beta \alpha}=0 \\
& d \theta_{\alpha \beta}=\sum_{\beta} \theta_{\alpha \gamma} \wedge \theta_{\gamma \beta} .
\end{aligned}
$$

Restricting these forms to the frames defined above, we have

$$
\theta_{3}=0
$$

and hence

$$
0=d \theta_{3}=\sum_{i} \theta_{i} \wedge \theta_{i 3} .
$$

By Cartan's lemma we may write

$$
\theta_{i 3}=\sum_{j} h_{i j} \theta_{j}, \quad h_{i j}=h_{j i} .
$$

The first equation of (1.3) gives

$$
d \theta_{i}=\sum_{j} \theta_{j} \wedge \theta_{j i}
$$

(where $\theta_{12}$ is the Levi-Civita connection form on $M$ which is uniquely determined by these two equations).

The Gauss equation is

$$
d \theta_{i j}=\sum_{k} \theta_{i k} \wedge \theta_{k j}+\Theta_{i j}
$$

where

$$
\begin{gathered}
\Theta_{12}=-\theta_{13} \wedge \theta_{23}=-\left(\operatorname{det}\left(h_{i j}\right)\right) \theta_{1} \wedge \theta_{2}, \\
K \stackrel{\text { def }}{=} \operatorname{det}\left(h_{i j}\right)=\text { the Gaussian curvature of } M .
\end{gathered}
$$

The Codazzi equations are

$$
d \theta_{i 3}=\sum_{j} \theta_{i j} \wedge \theta_{j 3} .
$$

The first fundamental form of the surface is

$$
\mathrm{I}=\left(\theta_{1}\right)^{2}+\left(\theta_{2}\right)^{2},
$$

and the second fundamental form is

$$
\mathrm{II}=\sum_{i} \theta_{i} \theta_{i 3}=\sum_{i j} h_{i j} \theta_{i} \theta_{j} .
$$

Near a non-umbilical point $M$ can be parametrized by its lines of curvature, i.e., there are coordinates $u_{i}$ in which I and II are both diagonalized. Explicitly we write

$$
\begin{aligned}
\mathrm{I} & =\left(a_{1}\right)^{2}\left(d u_{1}\right)^{2}+\left(a_{2}\right)^{2}\left(d u_{2}\right)^{2} \\
\mathrm{II} & =b_{1}\left(a_{1}\right)^{2}\left(d u_{1}\right)^{2}+b_{2}\left(a_{2}\right)^{2}\left(d u_{2}\right)^{2},
\end{aligned}
$$


so that $h_{i i}=b_{i}$ and $h_{12}=0$. Observe that $b_{i}$ are the two principal curvatures. The Riemannian connection is given by

$$
\theta_{12}=\frac{1}{a_{1}} \frac{\partial a_{2}}{\partial u_{1}} d u_{2}-\frac{1}{a_{2}} \frac{\partial a_{1}}{\partial u_{j}} d u_{1},
$$

and the Codazzi equation can be written

$$
\frac{1}{b_{i}-b_{j}} \frac{\partial b_{i}}{\partial u_{j}}=-\frac{\partial\left(\log a_{i}\right)}{\partial u_{j}}, i \neq j .
$$

Now suppose $M$ has constant negative curvature $K \equiv-1$; then $b_{1} b_{2}=-1$. By (1.15)

$$
\underset{b_{i}\left(b_{i}-b_{i}\right)}{\left.\partial b_{j}\right)} \frac{\partial b_{i}}{\partial u_{j}}=-\frac{\partial\left(\log a_{i}\right)}{\partial u_{j}}, i \neq j,
$$

or

$$
2 \frac{\partial\left(\log \left(b_{i}^{2}+1\right)\right)}{\partial u_{j}}=-\frac{\partial\left(\log a_{i}\right)}{\partial u_{j}}, i \neq j .
$$

Therefore there exist two positive valued functions $c_{1}\left(u_{1}\right), c_{2}\left(u_{2}\right)$ such that

$$
b_{i}^{2}+1=\frac{c_{i}\left(u_{i}\right)}{a_{i}^{2}} .
$$

Making a change in each coordinate separately, we may assume $c_{i}=1$. Writing $b_{1}=\tan \psi, b_{2}=-\cot \psi$, then $a_{1}=\cos \psi$ and $a_{2}=\sin \psi$, i.e.,

$$
\begin{aligned}
\mathbf{I} & =\cos ^{2} \psi\left(d u_{1}\right)^{2}+\sin ^{2} \psi\left(d u_{2}\right)^{2} \\
\mathrm{II} & =\sin \psi \cos \psi\left(\left(d u_{1}\right)^{2}-\left(d u_{2}\right)^{2}\right),
\end{aligned}
$$

so $2 \psi$ is the angle between the two asymptotic directions. The connection form (1.14) becomes

$$
\theta_{12}=\frac{\partial \psi}{\partial u_{1}} d u_{2}+\frac{\partial \psi}{\partial u_{2}} d u_{1} .
$$

The Gauss equation (1.8) then gives

$$
\frac{\partial^{2} \psi}{\partial\left(u_{1}\right)^{2}}-\frac{\partial^{2} \psi}{\partial\left(u_{2}\right)^{2}}=\sin \psi \cos \psi,
$$

i.e., $\phi=2 \psi$ satisfies the Sine-Gordon equation (SGE)

$$
\frac{\partial^{2} \phi}{\partial\left(u_{1}\right)^{2}}-\frac{\partial^{2} \phi}{\partial\left(u_{2}\right)^{2}}=\sin \phi .
$$

The converse is also true by the existence and uniqueness theorem on surfaces. Therefore we have proved: There is a one to one correspondence between solutions $\phi$ of SGE with $0<\phi<\pi$ and the local surfaces of constant Gaussian curvature $K \equiv-1$ in $\mathbf{R}^{3}$ up to rigid motion. 
A congruence of lines is an immersed surface in the Grassmann manifold $G r$ of all lines in $\mathbf{R}^{3}$. Locally we can suppose the lines be oriented, with their points given by

$$
Y=X(u, v)+\lambda \xi(u, v), \quad \xi^{2}=1,
$$

$\lambda$ being a parameter on each line. The equations

$$
u=u(t), v=v(t), u^{\prime 2}+v^{\prime 2} \neq 0
$$

define a ruled surface belonging to the congruence. It is a developable if and only if the determinant

$$
(\xi, d X, d \xi)=0 .
$$

This is a quadratic equation in $d u, d v$. Suppose that it has two real and distinct roots. There are then two families of developables, each of which (in the generic case) consists of the tangent lines of a surface. It follows that the lines of the congruence are the common tangent lines of two surfaces $M$ and $M^{*}$, to be called the focal surfaces. There results a mapping $\ell: M \rightarrow M^{*}$ such that the congruence consists of the lines joining $P \in M$ to $\ell(P) \in M^{*}$. This construction, of great geometrical simplicity, plays a fundamental role in the theory of transformation of surfaces.

Definition 1. Consider a line congruence with the focal surfaces $M, M^{*}$ such that its lines are the common tangents at $P \in M$ and $P^{*}=$ $\iota(P) \in M^{*}$. The congruence is called pseudo-spherical (p.s.) if

(1) $\left\|\mathbf{P P}^{*}\right\|=r$, which is a constant independent of $P$.

(2) The angle between the two normals $\nu_{P}$ and $\nu_{P} *$ at $P$ and $P^{*}$ is equal to a constant $\tau$ independent of $P$.

We can now state the classical Bäcklund theorem and give a simple proof by the method of moving frames.

THeOREM 1. Suppose there is a p.s. congruence in $\mathbf{R}^{3}$ with the focal surfaces $M$ and $M^{*}$ such that the distance $r$ between corresponding points and the angle $\tau$ between corresponding normals are constants. Then both $M$ and $M^{*}$ have constant negative Gaussian curvature equal to $-\sin ^{2} \tau / r^{2}$.

Proof. We choose an orthonormal frame $v_{1}, v_{2}, v_{3}$ on $M$ such that $v_{1}$ is the unit vector in the direction of $\mathbf{P P} *$ (where $P$ and $P^{*}$ are the corresponding points) and $v_{3}$ is the normal to $M$. From the definition of a p.s. congruence, there is an orthonormal frame $v_{1}^{*}, v_{2}^{*}, v_{3}^{*}$ on $M^{*}$ given by

$$
\begin{aligned}
& v_{1}^{*}=v_{1} \\
& v_{2}^{*}=\cos \tau v_{2}+\sin \tau v_{3} \\
& v_{3}^{*}=-\sin \tau v_{2}+\operatorname{con} \tau v_{3}=\text { the normal to } M^{*} .
\end{aligned}
$$


Suppose locally $M$ is given by an immersion $X: U \rightarrow \mathbf{R}^{3}$, where $U$ is an open subset of $\mathbf{R}^{2}$, then $M^{*}$ is given by

$$
X^{*}=X+r v_{1}
$$

Taking the differential of (1.24) gives

$$
\begin{aligned}
d X^{*} & =d X+r d v_{1} \\
& =\sum_{i} \theta_{i} v_{i}+r \sum_{\alpha} \theta_{1 \alpha} v_{\alpha} \\
& =\theta_{1} v_{1}+\left(\theta_{2}+r \theta_{12}\right) v_{2}+r \theta_{13} v_{3} .
\end{aligned}
$$

On the other hand, let $\theta_{i}^{*}$ be the dual coframe of $v_{i}^{*}$ we have

$$
\begin{aligned}
d X^{*} & =\theta_{1}^{*} v_{1}^{*}+\theta_{2}^{*} v_{2}^{*} \\
& =\theta^{*}{ }_{1} v_{1}+\theta_{2}^{*} \cos \tau v_{2}+\theta_{2}^{*} \sin \tau v_{3} .
\end{aligned}
$$

Comparing coefficients of $v_{i}$ in (1.25) and (1.26), we get

$$
\begin{aligned}
\theta_{1}^{*} & =\theta_{1} \\
\theta_{2}^{*} \cos \tau & =\theta_{2}+r \theta_{12} \\
\theta_{2}^{*} \sin \tau & =r \theta_{13}
\end{aligned}
$$

This gives

$$
\theta_{2}+r \theta_{12}=r \cot \tau \theta_{13} .
$$

Since $\theta_{1}^{*}, \theta_{2}^{*}$ are linearly independent, by (1.27) $h_{12}$ never vanishes. In order to compute the curvature of $M^{*}$, we have, by using (1.23), (1.28),

$$
\begin{aligned}
\theta_{13}^{*} & =d v_{1}^{*} \cdot v_{3}^{*} \\
& =-\sin \tau \theta_{12}+\cos \tau \theta_{13} \\
& =\frac{\sin \tau}{r} \theta_{2}, \\
\theta_{23}^{*} & =d v_{2}^{*} \cdot v_{3}^{*}=\theta_{23} .
\end{aligned}
$$

Then

$$
\begin{aligned}
\Theta_{12}^{*} & =-\theta_{13}^{*} \wedge \theta_{23}^{*} \\
& =-\frac{\sin \tau}{r} \theta_{2} \wedge \theta_{23} \\
& =\frac{\sin \tau}{r} h_{12} \theta_{1} \wedge \theta_{2} .
\end{aligned}
$$

On the other hand, using (1.27),

$$
\Theta_{12}^{*}=-K^{*} \theta_{1}^{*} \wedge \theta_{2}^{*}
$$




$$
=-K^{*} \frac{r}{\sin \tau} h_{12} \theta_{1} \wedge \theta_{2} .
$$

Comparing coefficients in (1 30) and (1.31), we have

$$
-K^{*} \frac{r h_{12}}{\sin \tau}=\frac{h_{12} \sin \tau}{r}
$$

Since $h_{12}$ never vanishes,

$$
K^{*}=-\frac{\sin ^{2} \tau}{r^{2}}
$$

By symmetry,

$$
K=-\frac{\sin ^{2} \tau}{r^{2}}
$$

The differential form equation (1.28) is called the Bäcklund transformation. We can write it as a system of partial differential equations. For simplicity, we may assume $K \equiv-1$, so that $r=\sin \tau$. Let $\alpha$ be the angle between the $u_{1}$-curves in the coordinate system (1.19) and the vector PP**. Then (1.28) becomes

$$
\begin{aligned}
& \frac{\partial(\alpha+\psi)}{\partial x}=a \sin (\alpha-\psi) \\
& \frac{\partial(\alpha-\psi)}{\partial y}=\frac{1}{a} \sin (\alpha+\psi),
\end{aligned}
$$

where $x=1 / 2\left(u_{1}+u_{2}\right), y=1 / 2\left(u_{1}-u_{2}\right)$ are the asymptotic coordinates, and $a=\csc \tau-\cot \tau=$ constant. In the asymptotic coordinates, the SGE becomes

$$
\frac{\partial^{2} \phi}{\partial x \partial y}=\sin \phi
$$

By equating the cross derivatives of (1.35), it follows that $2 \alpha$ is also a solution of SGE.

Note that the geometric derivation of (1.35) is local and the range of $\alpha$ and $\psi$ are restricted to lie in $(0, \pi / 2)$. However, from the analytic point of view, if $2 \psi$ is any solution of SGE and $\alpha, \psi$ satisfy (1.35) then $2 \alpha$ is also a solution.

Next we discuss the complete integrability of (1.28) or (1.35).

THEOREM 2. Suppose $M$ is a surface of constant negative curvature, $K=$ $-\sin ^{2} \tau / r^{2}$, where $r>0$ and $\tau$ are constants. Given any unit vector $v_{0} \in$ $T_{P_{0}}(M)$, which is not in a principal direction, there exists a unique surface $M^{*}$ and a p.s. congruence with $M$ and $M^{*}$ as focal surfaces such that if 
$P_{0}^{*} \in M^{*}$ is the point corresponding to $P_{0}$, we have $\mathbf{P}_{0} \mathbf{P}_{0}^{*}=r v_{0}$ and $\tau$ is the angle between the normals at $P_{0}, P_{0}^{*}$.

Proof. The differential form equation (1.28) is completely integrable, since

$$
\begin{aligned}
& d\left(\theta_{2}+r \theta_{12}-r \cot \tau \theta_{13}\right) \\
& \quad=\theta_{1} \wedge \theta_{12}+r \theta_{13} \wedge \theta_{32}-r \cot \tau \theta_{12} \wedge \theta_{23}, \\
& =\left(-\frac{1}{r}-r K \csc ^{2} \tau\right) \theta_{1} \wedge \theta_{2}, \text { using (1.28) } \\
& \quad=0, \text { since } K=-\frac{\sin ^{2} \tau}{r^{2}} .
\end{aligned}
$$

Then the theorem follows directly from Frobenius Theorem.

Definition 2. A line congruence $\ell: M \rightarrow M^{*}$ is called a Weingarten congruence (or $W$-congruence) if $\ell$ maps the asymptotic curves of $M$ to the asymptotic curves of $M^{*}$.

Corollary 1. A p.s. congruence is a W-congruence.

ProOF. II ${ }^{*}=\theta_{1}^{*} \theta_{13}^{*}+\theta_{2}^{*} \theta_{23}^{*}$

$$
\begin{aligned}
& =\frac{\sin \tau}{r} \theta_{1} \theta_{2}+\underset{\sin \tau}{r} \theta_{13} \theta_{23} \\
& =\frac{r h_{12}}{\sin \tau}\left[h_{11}\left(\theta_{1}\right)^{2}+2 h_{12} \theta_{1} \theta_{2}+h_{22}\left(\theta_{2}\right)^{2}\right], \text { using } K=\operatorname{det}\left(h_{i j}\right)=\frac{-\sin ^{2} \tau}{r^{2}} \\
& =\frac{r h_{12}}{\sin \tau} \text { II. }
\end{aligned}
$$

Since the second fundamental forms are proportional, the asymptotic curves correspond under $\ell$.

The above results generalize to the case of $n$-dimensional submanifolds of constant negative curvature in $\mathbf{R}^{2 n-1}$. This generalization will be the subject of a paper by K. Tenenblat and C. L. Terng.

In what follows we will study the analogue of the geometric Bäcklund transformation in affine differential geometry.

2. Affine surfaces. Let $A^{3}$ be the unimodular affine space of dimension 3 , i.e., the space with real coordinates $x^{1}, x^{2}, x^{3}$ and volume element $d V=d x^{1} \wedge d x^{2} \wedge d x^{3}$. The linear group $G$ which preserves the volume form is the unimodular affine group, i.e.,

$$
x^{* \alpha}=\sum_{\beta} c_{\beta}^{\alpha} x^{\beta}+d^{\alpha}
$$

where 


$$
\operatorname{det}\left(c_{\beta}^{\alpha}\right)=1
$$

Following $\S 1$ we shall continue to adopt the range 1, 2, 3, for small Greek indices and the range 1, 2 for small Latin indices. In the space $A^{3}$ distance and angle have no meaning, but there are the notions of vectors and parallelism.

Let $x$ be the position vector of the surface $M$ sitting in $A^{3}$. Let $x, e_{1}, e_{2}$, $e_{3}$ be an affine frame on $M$ such that $e_{1}, e_{2}$ are tangent to $M$ at $x$, and

$$
\left(e_{1}, e_{2}, e_{3}\right)=\operatorname{det}\left(e_{1}, e_{2}, e_{3}\right)=1 \text {. }
$$

We can write

$$
\begin{aligned}
d x & =\sum_{\alpha} \omega^{\alpha} e_{\alpha} \\
d e_{\alpha} & =\sum_{\beta} \omega_{\alpha}^{\beta} e_{\beta} .
\end{aligned}
$$

The $\omega^{\alpha}, \omega_{\alpha}^{\beta}$ are the Maurer-Cartan forms of $G$. Differentiating (2.3) and using (2.4), we get

$$
\sum_{\alpha} \omega_{\alpha}^{\alpha}=0
$$

The structure equation of $A^{3}$ gives

$$
\begin{aligned}
& d \omega^{\alpha}=\sum_{\beta} \omega^{\beta} \wedge \omega_{\beta}^{\alpha} \\
& d \omega_{\alpha}^{\beta}=\sum_{r} \omega_{\alpha}^{r} \wedge \omega_{r}^{\beta} .
\end{aligned}
$$

If we restrict the forms to the surface $M$ as defined above, we have

$$
\omega^{3}=0
$$

and the first equation of (2.6) gives

$$
\sum_{i} \omega^{i} \omega_{i}^{3}=0
$$

By Cartan's lemma, we have

$$
\omega_{i}^{3}=\sum_{k} h_{i k} \omega^{k}, h_{i k}=h_{k i} .
$$

From now on we assume that $M$ is non-degenerate, i.e., the rank of $\left(h_{i j}\right)$ is equal to 2 . Let $H=\operatorname{det}\left(h_{i j}\right)$. Then it follows that the quadratic differential form

$$
\mathrm{II}=|H|^{-\frac{1}{4}} \sum_{i, k} h_{i k} \omega^{i} \omega^{k}
$$

is affinely invariant. Since it is of rank 2 , it defines a pseudo-Riemannian structure on $M$, which is called the affine metric of $M$, to which the method 
of Riemannian geometry can be applied. In particular, the Gaussian curvature of II is defined.

Since $M$ is non-degenerate, we can choose $e_{3}$ suitably such that

$$
\omega_{3}^{3}+\frac{1}{4} d \log |H|=0 .
$$

Under such a choice the line through $x$ in the direction of $e_{3}$ is called the affine normal at $x$. Moreover, the vector $|H|^{1 / 4} e_{3}$ is affinely invariant, and is called the affine normal vector. The affine normal has interesting geometrical properties; see [1, 3].

Differentiating (2.11), we get

$$
\sum_{i} \omega_{3}^{i} \wedge \omega_{i}^{3}=0
$$

which gives

$$
\omega_{3}^{i}=\sum_{k} \ell^{i k} \omega_{k}^{3}, \quad \ell^{i k}=\ell^{k i} .
$$

Equation (2.13) can be written as

$$
\omega_{3}^{i}=\sum_{k} \ell_{k}^{i} \omega^{k},
$$

where

$$
\ell_{k}^{i}=\sum_{j} \ell^{i j} h_{j k} .
$$

Then we can verify easily that the quadratic differential form

$$
\mathrm{III}=\sum_{i} \omega_{3}^{i} \omega_{i}^{3}
$$

is invariant under any change of frame keeping the affine normal $e_{3}$ fixed. Therefore the trace of III relative to II, i.e.,

$$
L=\frac{1}{2}|H|^{\frac{1}{4}} \sum_{i} \ell_{i}^{i}
$$

is an affine invariant. It is called the affine mean curvature. $A$ surface is called affine minimal if $L=0$. These are the critical points for the variational problem for the affine area defined by II. (See [2]). Formula (2.18) can also be put in the form

$$
L \omega^{1} \wedge \omega^{2}=\frac{1}{2}|H|^{\frac{1}{4}}\left(\omega^{1} \wedge \omega_{3}^{2}+\omega_{3}^{1} \wedge \omega^{2}\right) .
$$

Next we define the Fubini-Pick cubic form of $M$. Taking the exterior differential of (2.9), we get

$$
\sum_{k}\left(d h_{i k}-\sum_{j} h_{i j} \omega_{k}^{j}-\sum h_{j k} \omega_{i}^{j}\right) \wedge \omega^{k}=0 .
$$


We define

$$
D h_{i k}=\sum_{j} h_{i k j} \omega^{j}=d h_{i k}-\sum_{j} h_{i j} \omega^{j}{ }_{k}-\sum_{j} h_{j k} \omega_{i}^{j} .
$$

It follows from (2.9) and (2.19) that $h_{i j k}$ are symmetric in all the indices. The cubic differential form

$$
P=\sum_{i, j, k} h_{i j k} \omega^{i} \omega^{j} \omega^{k}
$$

is called the Fubini-Pick form on M. It measures the difference between the affine connection

$$
D e_{i}=\omega_{i}^{j} e_{j}
$$

and the Levi-Civita connection of II, which we write as

$$
\tilde{D} e_{i}=\tilde{\omega}_{i}^{j} e_{j} \text {. }
$$

In fact, we find

$$
\tilde{\omega}_{i}^{j}=\omega_{i}^{j}+\frac{1}{2} \sum_{k, \iota} h^{j k} h_{i k \iota} \omega^{\prime},\left(h^{j k}\right)=\text { inverse matrix of }\left(h_{i k}\right) .
$$

Therefore the curvature tensor of the affine metric II can be computed from III and the Fubini-Pick form.

For later use, we will now develop the local theory for hyperbolic surfaces (i.e., $H<0$ ).

If $M$ is hyperbolic, we may assume $X(u, v)$ to be parametrized by its asymptotic curves. Choose $e_{1}=\partial x / \partial u, e_{2}=\partial x / \partial v$, and $e_{3}$ to be in the affine normal direction such that

$$
\left(e_{1}, e_{2}, e_{3}\right)=1
$$

Then

$$
\omega^{1}=d u, \omega^{2}=d v, \omega_{1}^{3}=h_{12} d v, \omega_{2}^{3}=h_{12} d u,
$$

and by supposing $h_{12}>0$,

$$
\omega_{3}^{3}=-\frac{1}{2} d \log h_{12} .
$$

The affine metric is

$$
\mathrm{II}=2 F d u d v
$$

where $F=\left(h_{12}\right)^{1 / 2}$.

Then a standard computation implies that the Gaussian curvature of II, which is called the affine curvature is given by

$$
\tilde{K}=-\frac{1}{F} \frac{\partial^{2} \log F}{\partial u \partial v} .
$$


By $(2.20)$

$$
\begin{aligned}
& h_{12 j} \omega^{j}=d h_{12}-h_{12} \omega_{2}^{2}-h_{12} \omega_{1}^{1}+h_{12} \omega_{3}^{3}=0 \\
& h_{11 j} \omega^{j}=-2 h_{12} \omega_{1}^{2}, \\
& h_{22 j} \omega^{j}=-2 h_{12} \omega_{2}^{1} .
\end{aligned}
$$

So we have

$$
\begin{aligned}
h_{12 j} & =0, \\
\omega_{1}^{2} & =-\frac{h_{111}}{2 h_{12}} d u, \\
\omega_{2}^{1} & =-\frac{h_{222}}{2 h_{12}} d v .
\end{aligned}
$$

Therefore the Fubini-Pick form is

$$
P=h_{111}(d u)^{3}+h_{222}(d v)^{3} .
$$

Using the structure equation (2.6), we get

$$
\begin{aligned}
\omega_{1}^{1} & =\frac{\partial}{\partial u}(\log F) d u \\
\omega_{2}^{2} & =\frac{\partial}{\partial v}(\log F) d v \\
\omega_{3}^{1} & =\ell d u+\ell_{2}^{1} d v \\
\omega_{3}^{2} & =\ell_{1}^{2} d u+\ell d v, \quad \ell=\ell_{1}^{1}=\ell_{2}^{2}
\end{aligned}
$$

where

$$
\begin{aligned}
\ell_{1}^{2} & =-\frac{1}{F^{3}} \frac{\partial}{\partial v}\left(\frac{h_{111}}{2 F}\right), \\
\ell_{2}^{1} & =-\frac{1}{F^{3}} \frac{\partial}{\partial u}\left(\frac{h_{222}}{2 F}\right), \\
L & =F \iota=J-K, \\
J & =\frac{h_{111} h_{222}}{4 F^{5}} .
\end{aligned}
$$

Since $\tilde{K}, L$ are affine invariants, $J$ is also an affine invariant.

Next we develop a necessary and sufficient condition for a graph to be affine minimal. Let a surface be locally given by

$$
x^{3}=f\left(x^{1}, x^{2}\right)
$$

So $x=\left(x^{1}, x^{2}, f\left(x^{1}, x^{2}\right)\right)$ is the position vector. Then equations (2.4), (2.5) hold if we set 


$$
\begin{aligned}
& \omega^{i}=d x^{i} \\
& \omega^{3}=d x^{3}-\frac{\partial f}{\partial x^{1}} d x^{1}-\frac{\partial f}{\partial x^{2}} d x^{2} \\
& e_{1}=\left(1,0, \frac{\partial f}{\partial x^{1}}\right) \\
& e_{2}=\left(0,1, \frac{\partial f}{\partial x^{2}}\right) \\
& e_{3}=(0,0,1)
\end{aligned}
$$

with

$$
\begin{aligned}
\omega_{i}^{j} & =0 \\
\omega_{i}^{3} & =\sum_{j} \frac{\partial^{2} f}{\partial x^{i} \partial x^{j}} \omega^{j} .
\end{aligned}
$$

Hence $h_{i j}=\partial^{2} f / \partial x^{i} \partial x^{j}$ and $H=$ Hessian of $f$. To find the affine normal, we let

$$
\begin{aligned}
& e_{i}^{*}=e_{i} \\
& e_{3}^{*}=e_{3}+a^{1} e_{1}+a^{2} e_{2}
\end{aligned}
$$

where $e_{3}^{*}$ is in the affine normal direction. Then $a_{i}$ 's are determined by

$$
d \log |H|+4 \sum_{i, k} a^{i} h_{i k} d x^{k}=0 .
$$

Hence

$$
a^{i}=-\sum_{j} \frac{h^{i j}}{4} \frac{\partial}{\partial x^{j}}(\log |H|)
$$

where

$$
\left(h^{i j}\right)=\left(h_{i j}\right)^{-1} .
$$

We compute

$$
\begin{aligned}
& \omega_{3}^{* 1}=\left(d e_{3}^{*}, e_{2}^{*}, e_{3}^{*}\right)=d a^{1}+\frac{a^{1}}{4} d \log |H| \\
& \omega_{3}^{* 2}=\left(e_{1}^{*}, d e_{3}^{*}, e_{3}^{*}\right)=d a^{2}+\frac{a^{2}}{4} d \log |H| .
\end{aligned}
$$

Therefore the affine mean curvature is

$$
\begin{aligned}
L & =\frac{1}{2}|H|^{\frac{1}{4}} \sum_{i, j}\left\{\frac{3 h^{i j}}{16} \frac{\partial}{\partial x^{i}}(\log |H|) \frac{\partial}{\partial x^{j}}(\log |H|)\right. \\
& \left.-\frac{h^{i j}}{4} \frac{\partial^{2}}{\partial x^{i} \partial x^{j}}(\log |H|)\right\} .
\end{aligned}
$$


We note that the equation for affine minimal surfaces is a fourth order equation in $f$.

If $f$ is a non-degenerate quadratic polynomial, then $H=$ constant. Hence the elliptic paraboloid $x^{3}=\left(x^{1}\right)^{2}+\left(x^{2}\right)^{2}$ and the hyperbolic paraboloid $x^{3}=\left(x^{1}\right)^{2}-\left(x^{2}\right)^{2}$ are affine minimal surfaces.

Our next result is a formula for affine mean curvature in terms of Riemannian geometry.

Let $e_{1}, e_{2}, e_{3}$ be a local orthonormal frame field on $M$ such that $e_{1}, e_{2}$ are tangent to $M, \theta_{i}$ is the dual coframe and $\theta_{\alpha \beta}$ are defined by

$$
d e_{\alpha}=\sum_{\beta} \theta_{\alpha \beta} e_{\beta}, \theta_{\alpha \beta}+\theta_{\beta \alpha}=0 .
$$

Then we have equations (1.1) and (1.4) as in section 1, and

$$
H=\operatorname{det}\left(h_{i j}\right)=K=\text { Gaussian curvature of } M \text {. }
$$

To find the affine normal direction, we let

$$
\begin{aligned}
& e_{i}^{*}=e_{i} \\
& e_{3}^{*}=e_{3}+a^{1} e_{1}+a^{2} e_{2},
\end{aligned}
$$

where $e_{3}^{*}$ is in the affine normal direction, then $a^{i}$ s are determined by

$$
d \log |K|+4 \sum_{i} a^{i} h_{i k} \omega^{k}=0,
$$

Hence

$$
a^{i}=-\sum \frac{h^{i j}}{4}(\log |K|)_{j},
$$

where $(f)_{j}$ denotes the covariant derivative of $f$ with respect to $e_{j}$. In fact

$$
\begin{aligned}
& D f=d f=\sum_{i} f_{i} \omega^{i} \\
& D f_{i}=\sum_{j} f_{i j} \omega^{j}=d f_{i}-\sum_{j} f_{j} \omega_{i}^{j},
\end{aligned}
$$

We compute

$$
\begin{aligned}
\omega_{3}^{* 1} & =\left(d e_{3}^{*}, e_{2}^{*}, e_{3}^{*}\right)=\omega_{3}^{1}+D a^{1}+\frac{a^{1}}{4} d \log |K|, \\
\omega_{3}^{* 2} & =\left(e_{1}^{*}, d e_{3}^{*}, e_{3}^{*}\right)=\omega_{3}^{2}+D a^{2}+\frac{a^{2}}{4} d \log |K|, \\
L= & { }_{2}^{1}|K|^{\frac{1}{4}}\left\{-\left(h_{11}+h_{22}\right)\right. \\
& \left.-\sum \frac{h^{i j}}{4}(\log |K|)_{i j}+\frac{3}{16} \sum h^{i j}(\log |K|)_{i}(\log |K|)_{j}\right\} .
\end{aligned}
$$

One immediate application of this formula is the following theorem 
TheOREm 3. Suppose $M$ is a surface in $\mathbf{R}^{3}$ which is isometric to a piece of the elliptic paraboloid with its induced Riemannian metric. Then as an affine surface $M$ is affine minimal.

Proof. Rewrite (2.50) as follows

$$
\begin{aligned}
2|K|^{-\frac{1}{4}} L & =h_{11}\left\{-K-\frac{1}{4}(\log |K|)_{22}+\frac{3}{16}(\log |K|)_{2}^{2}\right\} \\
& -2 h_{12}\left\{-\frac{1}{4}(\log |K|)_{12}+\frac{3}{16}(\log |K|)_{1}(\log |K|)_{2}\right\} \\
& +h_{22}\left\{-K-\frac{1}{4}(\log |K|)_{11}+\frac{3}{16}(\log |K|)_{1}^{2}\right\} .
\end{aligned}
$$

(In this formula the superscript 2 means square.)

For the surface $x^{3}=\left(x^{1}\right)^{2}+\left(x^{2}\right)^{2}$, we choose coordinates

$$
X(u, v)=\left(v \cos u, v \sin u, v^{2}\right) .
$$

Then the coefficients of $h_{i j}$ in (2.51) vanish identically, and the theorem follows from the fact that these coefficients only depend on the first fundamental form of the surface.

3. Bäcklund theorem for affine surfaces. In this section we are going to prove our main theorem:

THEOREM 4. Let $M$ and $M^{*}$ be the focal surfaces of a $W$-congruence in $A^{3}$, with the correspondence denoted by $\iota: M \rightarrow M^{*}$ such that the affine normals at $P$ and $P^{*}=\ell(P)$ are parallel. Then both $M$ and $M^{*}$ are affine minimal surfaces.

Proof. Choose an affine frame $e_{1}, e_{2}, e_{3}$ such that

$$
e_{1}=\overrightarrow{\mathbf{P P}} *
$$

$e_{2}$ is tangent to $M$ at $P$

$e_{3}$ is in the affine normal direction.

Suppose the position vector for $M$ is given by $X$. Then the position vector for $M^{*}$ is given by

$$
X^{*}=X+e_{1} .
$$

There exists a function $k$ such that

$$
\begin{aligned}
& e_{1}^{*}=-e_{1} \\
& e_{2}^{*}=e_{2}+\frac{1}{k} e_{3} \\
& e_{3}^{*}=-e_{3}
\end{aligned}
$$


is an affine frame on $M^{*}$, with $e_{2}^{*}$ tangent to $M^{*}$ at $X^{*}$. Let $\omega^{* i}$ be the dual frame of (3.3). Then

$$
\begin{aligned}
d X^{*} & =\omega^{* 1} e_{1}^{*}+\omega^{* 2} e_{2}^{*} \\
& =-\omega^{* 1} e_{1}+\omega^{* 2}\left(e_{2}+\frac{1}{k} e_{3}\right) .
\end{aligned}
$$

However, differentiating (3.2) we get

$$
\begin{aligned}
d X^{*} & =d X+d e_{1} \\
& =\left(\omega^{1}+\omega_{1}^{1}\right) e_{1}+\left(\omega^{2}+\omega_{1}^{2}\right) e_{2}+\omega_{1}^{3} e_{3} .
\end{aligned}
$$

Comparing coefficients of (3.4) and (3.5), we get

$$
\begin{aligned}
\omega^{* 1} & =-\left(\omega^{\mathrm{I}}+\omega_{1}^{1}\right) \\
\omega^{* 2} & =\omega^{2}+\omega_{1}^{2} \\
\frac{1}{k} \omega^{* 2} & =\omega_{1}^{3} .
\end{aligned}
$$

Hence

$$
\omega^{2}+\omega_{1}^{2}=k \omega_{1}^{3} .
$$

Let

$$
\begin{aligned}
& \alpha=\omega^{1}+\omega_{1}^{1}+k \omega_{2}^{3} \\
& \beta=-\omega_{3}^{2}-d k+2 k \omega_{3}^{3} \\
& \gamma=\omega^{2}+\omega_{1}^{2}-k \omega_{1}^{3}=0 .
\end{aligned}
$$

Then we have

$$
\begin{aligned}
& \omega^{* 1}=k \omega_{3}^{3}-\alpha \\
& \omega^{* 2}=k \omega_{1}^{3} .
\end{aligned}
$$

From (2.4) we have

$$
\begin{aligned}
\omega_{1}^{* 3} & =\left(e_{1}^{*}, e_{2}^{*}, d e_{1}^{*}\right) \\
& =\left(-e_{1}, e_{2}+\frac{1}{k} e_{3},-d e_{1}\right) \\
& =\omega_{1}^{3}-\frac{1}{k} \omega_{1}^{2} \\
& =\frac{1}{k} \omega^{2}, \quad \text { using (3.7). }
\end{aligned}
$$

Similarly, we have

$$
\omega_{2}^{* 3}=-\frac{1}{k^{2}}(k \alpha+\beta)+\frac{1}{k} \omega^{1}
$$




$$
\begin{aligned}
& \omega_{3}^{* 3}=\omega_{3}^{3}-\frac{1}{k} \omega_{3}^{2} \\
& \omega_{3}^{* 1}=\omega_{3}^{1} \\
& \omega_{3}^{* 2}=-\omega_{3}^{2} .
\end{aligned}
$$

It follows from (2.18a), (3.9a), (3.9b) and (3.9c) that

$$
\begin{aligned}
L^{*} \omega^{* 1} \wedge \omega^{* 2} & =\frac{1}{2}\left|H^{*}\right|^{\frac{1}{4}}\left(\omega^{* 1} \wedge \omega_{3}^{* 2}+\omega_{3}^{* 1} \wedge \omega^{* 2}\right) \\
& =\frac{1}{2}\left|H^{*}\right|^{\frac{1}{4}}\left[\left(-k \omega_{2}^{3}+\alpha\right) \wedge \omega_{3}^{2}+k \omega_{3}^{1} \wedge \omega_{1}^{3}\right] \\
& =\frac{1}{2}\left|H^{*}\right|^{\frac{1}{4}}\left[k\left(\omega_{3}^{1} \wedge \omega_{1}^{3}+\omega_{3}^{2} \wedge \omega_{2}^{3}\right)+\alpha \wedge \omega_{3}^{2}\right] \\
& =\frac{1}{2}\left|H^{*}\right|^{\frac{1}{4}}\left[k d \omega_{3}^{3}+\alpha \wedge \omega_{3}^{2}\right] .
\end{aligned}
$$

Since $e_{3}$ is in the affine normal direction, $d \omega_{3}^{3}=0$ by (2.11). So we have

$$
L^{*} \omega^{* 1} \wedge \omega^{* 2}=\frac{1}{2}\left|H^{*}\right|^{\frac{1}{4}} \alpha \wedge \omega_{3}^{2} .
$$

By hypothesis, $e_{3}$ and $e_{3}^{*}$ are in the affine normal directions of $M$ and $M^{*}$ respectively; we rewrite (2.11) as

$$
\begin{gathered}
\omega_{3}^{3}=-\frac{1}{4} d \log |H| \\
\omega_{3}^{* 3}=-\frac{1}{4} d \log \left|H^{*}\right|
\end{gathered}
$$

Next we compute the following tensor by using (3.9a), (3.9b) and (3.9c) getting,

$$
\begin{aligned}
\sum_{i, j} h_{j j}^{*} \omega^{* i} \otimes \omega^{* j} & =\sum_{i} \omega^{* i} \otimes \omega_{i}^{* 3} \\
& =\left(k \omega_{2}^{3}-\alpha\right) \otimes\left(\frac{1}{k} \omega^{2}\right)+k \omega_{1}^{3} \otimes\left[-\frac{1}{k^{2}}(k \alpha+\beta)+\frac{1}{k} \omega^{1}\right] \\
& =\omega_{2}^{3} \otimes \omega^{2}+\omega_{1}^{3} \otimes \omega^{1}-\frac{1}{k}\left[\alpha \otimes \omega^{2}+\omega_{1}^{3} \otimes(k \alpha+\beta)\right] \\
& =\sum_{i, j} h_{i j} \omega^{i} \otimes \omega^{j}-\frac{1}{k}\left[\alpha \otimes \omega^{2}+\omega_{1}^{3} \otimes(k \alpha+\beta)\right] .
\end{aligned}
$$

We note that the tensors $\sum_{i, j} h_{i j}^{*} \omega^{* i} \otimes \omega^{* j}$ and $\sum_{i, j} h_{i j} \omega^{i} \otimes \omega^{j}$ are symmetric and the same must be true of their difference. Because $\ell$ is a $W$ congruence (i.e., II* is a multiple of II), these two tensors are proportional in the tensor space. Hence there exists a function $b$ such that 


$$
\alpha \otimes \omega^{2}+\omega_{1}^{3} \otimes(k \alpha+\beta)=b \sum_{i, j} h_{i j} \omega^{i} \otimes \omega^{j} .
$$

This $b \neq k$, for otherwise $\mathrm{II}^{*}=0$, contradicting the non-degeneracy of $M^{*}$.

Suppose

$$
\begin{aligned}
\alpha & =a_{1} \omega^{1}+a_{2} \omega^{2} \\
k \alpha+\beta & =b_{1} \omega^{1}+b_{2} \omega^{2} .
\end{aligned}
$$

Comparing the coefficients of $\omega^{i} \otimes \omega^{j}$ in (3.13), we get

$$
\begin{aligned}
a_{1}+h_{11} b_{2} & =h_{12} b_{1}=h_{12} b \\
h_{11} b_{1} & =h_{11} b \\
a_{2}+h_{12} b_{2} & =h_{22} b .
\end{aligned}
$$

Since $M$ is non-degenerate, $h_{11}$ and $h_{12}$ cannot vanish simultaneously, and (3.15), (3.16) imply that $b_{1}=b$. It also follows from (3.15) and (3 17) that

$$
a_{1} h_{12}-a_{2} h_{11}=-b H .
$$

Using (3.9) and (3.18), we get

$$
\begin{aligned}
& \omega^{* 1} \wedge \omega^{* 2}=k H(b-k) \omega^{1} \wedge \omega^{2} \\
& \omega_{1}^{* 3} \wedge \omega_{2}^{* 3}=\frac{1}{k^{3}}(b-k) \omega^{1} \wedge \omega^{2} .
\end{aligned}
$$

However,

$$
\omega_{1}^{* 3} \wedge \omega_{2}^{* 3}=H^{*} \omega^{* 1} \wedge \omega^{* 2}
$$

and $(b-k)$ never vanishes, so we have

$$
k^{4} H^{*} H=1 \text {. }
$$

Taking $1 / 4 d \log$ of (3.22) and using (3.11), we obtain

$$
d k-\omega_{3}^{* 3}-\omega_{3}^{3}=0 .
$$

Then (3.9) implies that

$$
\beta=0 .
$$

Therefore by (3.14)

$$
b_{i}=k a_{i} .
$$

Substituting (3.24) in (3.15) and (3.17), we obtain

$$
\begin{aligned}
& \left(1-k h_{12}\right) a_{1}+h_{11} k a_{2}=0 \\
& -h_{22} a_{1}+\left(1+k h_{12}\right) a_{2}=0 .
\end{aligned}
$$


The determinant of $(3.25)$ is

$$
k^{2} H+1 .
$$

If $k^{2} H+1 \neq 0$ then $a_{i}=0$, so $\alpha=0$. And if $k^{2} H+1 \equiv 0$, then

$$
\frac{d k}{2 k}-\omega_{3}^{3}=0 .
$$

But $\beta=0$, so $\omega_{3}^{2}=0$. Therefore we have shown that either $\alpha$ or $\omega_{3}^{2}$ is zero, so by (3.10) $L^{*}=0$. Then by symmetry $L=0$, i.e., both $M$ and $M^{*}$ are affine minimal.

We use the same notations as in the proof of the above theorem. We claim that if $\omega_{3}^{2}=0$ then $\overrightarrow{\mathbf{P P}}^{*}$ is an asymptotic vector. Indeed using (3.9), $\omega_{3}^{2}=0, L=0$, and $L^{*}=0$, we have

$$
\begin{aligned}
\omega_{3}^{* 1} & =\ell_{2}^{* 1} \omega^{* 2}=k \ell_{2}^{* 1} \omega_{1}^{3} \\
& =\omega_{3}^{1}={ }_{2}^{1} \omega^{2} .
\end{aligned}
$$

Therefore $h_{11} \equiv 0$, i.e., $e_{1}=\overrightarrow{\mathbf{P P}}^{*}$ is an asymptotic vector.

Suppose $\overrightarrow{\mathbf{P P}}^{*}$ is an asymptotic vector for all $P$, then $h_{11} \equiv 0$. By using the local theory for hyperbolic affine surfaces in section 2 and (3.7), we can conclude that $\omega_{1}^{2}=0$ and $k=1 / h_{12}$. By (2.28), we have $h_{111}=0$. So $J=0$. But we have already shown that $L=0$, hence $\tilde{K}=0$. Therefore we have proved the following two corollaries.

Corollary 1. Assumptions as in Theorem 4. If $\overrightarrow{\mathbf{P P}} *$ is not in the asymptotic direction for all $P \in M$, then

$$
\alpha=0, \beta=0, \gamma=0 .
$$

CoRollary 2. Assumptions as in Theorem 4. If $\overrightarrow{\mathbf{P P}}^{*}$ is an asymptotic vector for all $P \in M$, then both $M$ and $M^{*}$ are affine minimal and affinely flat (i.e., the affine curvature is zero).

Now we wish to prove the integrability theorem.

THeOREM 5. Suppose $M$ is an affine minimal surface in $A^{3}$. Given $v_{0} \in$ $T_{P_{0}}(M)$ which is not an asymptotic vector, then there exist a surface $M^{*}$ and a $W$-congruence $\ell: M \rightarrow M^{*}$ with parallel affine normals at $P \in M$ and $P^{*}=\ell(P) \in M^{*}$ and $\overrightarrow{\mathbf{P}_{0} \mathbf{P}_{0}} *=v_{0}$.

Proof. Taking the differential of the system (3.28), we have

$$
\begin{aligned}
& d \alpha=\gamma \wedge \omega_{2}^{1}-\beta \wedge \omega_{2}^{3}+\alpha \wedge \omega_{1}^{1} \\
& d \beta=-\omega_{3}^{1} \wedge \gamma+\omega_{3}^{2} \wedge \alpha+2 \beta \wedge \omega_{1}^{3}-2|H|^{-\frac{1}{4}} L \omega^{1} \wedge \omega^{2} \\
& d \gamma=\alpha \wedge \omega_{1}^{2}-\omega_{1}^{3} \wedge \beta .
\end{aligned}
$$


That the system (3.28) is completely integrable follows from the fact that $M$ is affine minimal. So there exist a function $k$ and an affine frame $e_{1}$, $e_{2}, e_{3}$ with $e_{3}$ in the affine normal direction and $e_{1}\left(P_{0}\right)=v_{0}$ such that $\alpha=$ $0, \beta=0, \gamma=0$.

Let $X$ be the position vector of $M$ in $A^{3}$, and $X^{*}=X+e_{1}$. Using $r=0$, we have

$$
\begin{aligned}
d X^{*} & =d X+d e_{1} \\
& =\left(\omega^{1}+\omega_{1}^{1}\right) e_{1}+k \omega_{1}^{3}\left(e_{2}+\frac{1}{k} e_{3}\right) .
\end{aligned}
$$

Since $\alpha=0$,

$$
\begin{aligned}
\left(\omega^{1}+\omega_{1}^{1}\right) \wedge k \omega_{1}^{3} & =k^{2} \omega_{1}^{3} \wedge \omega_{2}^{3} \\
& =k^{2} H \omega^{1} \wedge \omega^{2} .
\end{aligned}
$$

Since $M$ is non-degenerate, $\omega^{1}+\omega_{1}^{1}$ and $k \omega_{1}^{3}$ are linearly independent. Hence $X^{*}$ defines a surface $M^{*}$ having $e_{1}, e_{2}+1 / k e_{3}$ as tangent at $X^{*}$. Therefore we can choose an affine frame on $M^{*}$ as follows

$$
\begin{aligned}
& e_{1}^{*}=-e_{1} \\
& e_{2}^{*}=e_{2}+\frac{1}{k} e_{3} \\
& e_{3}^{*}=-e_{3} .
\end{aligned}
$$

Then we have (3.9). Since $\alpha=\beta=0$, (3.12) implies that

$$
\left|H^{*}\right|^{\frac{1}{4}} \mathrm{II}^{*}=|H|^{\frac{1}{4}} \mathrm{II},
$$

i.e., $\ell: X \mapsto X^{*}$ is a $W$-congruence.

Next we want to show that $e_{3}^{*}$ is in the direction of affine normal of $M^{*}$. By (3.9),

$$
\omega_{1}^{* 3} \wedge \omega_{2}^{* 3}=\frac{-1}{k^{2}} \omega^{1} \wedge \omega^{2} .
$$

However,

$$
\begin{aligned}
\omega_{1}^{* 3} \wedge \omega_{2}^{* 3} & =H^{*} \omega^{* 1} \wedge \omega^{* 2} \\
& =-k^{2} H^{*} H \omega^{1} \wedge \omega^{2} \operatorname{using}(3.9),
\end{aligned}
$$

so

$$
k^{4} H^{*} H=1 .
$$

Since $e_{3}$ is in the affine normal direction, $\omega_{3}^{3}=-1 / 4 d \log |H|$. By (3.36), we have 


$$
\frac{d k}{k}+\frac{d \log \left|H^{*}\right|}{4}-\omega_{3}^{3}=0 .
$$

Using (3.9), (3.37) and $\beta=0$, we get

$$
\begin{aligned}
\omega_{3}^{* 3} & =\omega_{3}^{3}-\frac{1}{k} \omega_{3}^{2} \\
& =\frac{d k}{k}-\omega_{3}^{3} \\
& =-\frac{1}{4} d \log \left|H^{*}\right|,
\end{aligned}
$$

i.e., $e_{3}^{*}$ is in the affine normal direction of $M^{*}$.

We note that if $M$ is affinely flat and affine minimal in $A^{3}$ with position vector $X$, then given any asymptotic vector field $e_{1}$ on $M$ such that $X^{*}=$ $X+e_{1}$ defines a surface in $A^{3}$, it follows from the local theory for hyperbolic surfaces in section 2 that $\ell: X \rightarrow X^{*}$ defines a $W$-congruence with parallel affine normals.

The significance of the above theorem in geometry is that we can construct new affine minimal surfaces by solving the completely integrable system (3.28) on a given affine minimal surface. This fact seems to be of some geometric interest. We do not know, however, whether it has any physical applications.

Our theorem can most likely be generalized to higher dimensions.

\section{REFERENCES}

1. W. Blaschke, Vorlesungen über Differential geometrie II, Berlin 1923.

2. S. S. Chern, Affine minimal hypersurfaces, Proceedings of US-Japan Seminar on Minimal Submanifolds, Tokyo 1978, 1-14.

3. P. A. Schirokow and A. P. Schirokow, Affine Differentialgeometrie, Leipzig 1962 (translated from Russian).

University of CALIfornia, Berkeley CA 94720. 